\title{
Preventive and Therapeutic Effects of Quercetin on Experimental Radiation Induced Lung Injury in Mice
}

\author{
Juan Wang ${ }^{1,2 \&}$, Yuan-Yuan Zhang ${ }^{3 \&}$, Jian Cheng ${ }^{4}$, Jing-Ling Zhang ${ }^{5}$, Bao-Sheng Li ${ }^{1,2,3 *}$
}

\begin{abstract}
Objective: To investigate the protective effect of quercetin on radiation induced lung injury (RILI) and related mechanisms. Materials and Methods: Mice treated with radiation and/or quercetin were sacrificed at 1-8 weeks after irradiation under anesthesia. Lung tissues were collected for histological examination. Immunohistochemistry (IHC) and Western blotting were performed to detect the protein expression of nuclear factor- $\kappa B(N F-\kappa B)$ and Mitogen-activated protein kinases (MAPK) pathway. Results: Hematoxylin and eosin (HE) staining showed that radiation controls displayed more severe lung damage than quercetin groups, either high or low dose. Results of IHC and Western blotting demonstrated the expression level of NF- $\mathrm{KB}$ to be decreased and that of an inhibitor of NF- $\kappa B$ (I $\kappa b-\alpha)$ to be increased by the quercetin intervention compared with the radiation control group. Numbers of JNK/SAPK, p38 and p44/p42 positive inflammatory cells were decreased in the radiation+quercetin injection group $(P<0.05)$. Conclusions: Quercetin may play a radio-protective role in mice lung via suppression of NF- $\mathrm{KB}$ and MAPK pathways.
\end{abstract}

Keywords: Quercetin - RILI - NF- $x$ B - MAPK

Asian Pac J Cancer Prev, 16 (7), 2909-2914

\section{Introduction}

Radiation therapy (RT) has been the main approach for primary lung carcinoma. However, its application is greatly limited because of radiation induced lung injury (RILI), which occurs in approximately $10-20 \%$ of patients undergoing radiotherapy (Mehta et al., 2005). RILI is not only one of the main obstacles to the dose escalation of radiotherapy but also affecting the treatment and even the post-radiation quality of life due to the symptoms caused by RILI like cough, fever, shortness of breath or even death. Thus, development of new therapies or strategies to attenuate RILI is critical to improve clinical outcome of lung cancer patients.

Evidence has suggested inflammation played a central role in the initiation and establishment of RILI (Ward et al., 1998), especially for the acute RILI, which is characterized by radiation pneumonitis 1-8 weeks post radiation. Therefore, signal pathways involved in inflammation may play a part in the development of RILI. NF- $x$ B and MAPK pathways can both be activated by $\mathrm{UV}$ or radiation; they perform as key transcription factors for pro-inflammatory mediators such as iNOS, TNF- $\alpha$, and interleukin-1 $\beta$ (IL-1 $\beta$ ), which can then activate macrophages. Activated macrophages produce many cytokines, tumor necrosis factor- $\alpha$ (TNF- $\alpha)$, interleukin- 6
(IL-6) and other inflammatory mediators such as nitric oxide (NO) and prostaglandin E2 (PGE2) (Reddy et al., 2009). Therefore, inhibitor of NF- $x$ B or MAPK may have certain effect in preventing or treating the RILI.

Quercetin is a member of flavonoids family derived from plants that play several pharmacological activities like anti-viral, anti-microbial, anti-inflammatory and anti-allergic potential (Chirumbolo et al., 2010). Like many other molecules sharing a flavone ring, quercetin affects immunity and inflammation through acting mainly on leukocytes and targeting many intracellular signaling kinases and phosphatases, enzymes and membrane proteins. Several recent published articles have reported protective effects of quercetin against radiation induced damage of human cells (Das et al., 2013), lymphocytes (Materska et al., 2015), skin of mice (Horton et al., 2013) and kidney and bladder tissues of rats (Ozyurt et al., 2014). Therefore, we designed this study to investigate the radioprotective effect of quercetin on RILI of mice lung and its underlying mechanism.

\section{Materials and Methods}

\section{Reagents}

Quercetin was obtained from Sigma-Aldrich and dissolved according to manufacturer's instructions to the

${ }^{I}$ Key Laboratory of Cancer Prevention and Therapy, Tianjin Medical University Cancer Institute and Hospital, Tianjin, ${ }^{2}$ Department of Radiation Oncology, Shandong Academy of Medical Sciences, ${ }^{3}$ Department of Radiation Oncology, Shandong Cancer Hospital, School of Medicine and Life Sciences, University of Jinan-Shandong Academy of Medical Scientists ${ }^{4}$ the Second Hospital of Shandong University, ${ }^{5}$ Linyi People's Hospital, Affiliated Hospital of Shandong University, Shandong, China ${ }^{\star}$ Equal contributors *For correspondence: baoshli1963@163.com 
final concentration of $2 \mathrm{mg} / \mathrm{ml}$ (low concentration) and 4 $\mathrm{mg} / \mathrm{ml}$ (high concentration) separately. Amifostine was obtained from Nanjing Kanghai Pharmaceutical Company, and was administrated through tail vein injection with the concentration of $4 \mathrm{mg} / \mathrm{ml}$ and total dose of $20 \mathrm{mg} / \mathrm{Kg}$. Rabbit polyclonal anti-NF- $x$ B p65 anti-P38, anti-SAPKJNK and anti-I $\varkappa$ B- $\alpha$ and anti-P44/P42 antibody were purchased from Cell Signaling Technology company (USA).

\section{Animals and irradiation}

80 female mice (BABL/C, 8 weeks old, weighing 19$21 \mathrm{~g}$ ) were purchased from the Shandong Experimental Animal Center and maintained at an animal facility. Mice were anesthetized before RT with intraperitoneal injection of pentobarbital $(40 \mathrm{mg} / \mathrm{kg}$ ) and were placed in a specially designed restrainer with adhesive tapes. The procedure of irradiation was the same as our previous study (Wang et al., 2012). The whole thorax of mice was exposed to a single dose of 10Gy with the assistance of lead blocks protecting the rest of the body.

\section{Grouping and treatment for mice}

These mice were randomly divided into 6 groups: 1$)$ normal control (8 mice), 100ul normal saline (NS) was administrated through tail vein 30 minutes before mock radiation, and then twice a week during the subsequent 6 weeks; 2) quercetin control (8 mice), 100ul quercetin (4 $\mathrm{mg} / \mathrm{ml}$ ) was administrated through tail vein 30 minutes before mock radiation, and then twice a week during the subsequent 6 weeks; 3 ) radiation control (16 mice), a single dose of $10 \mathrm{~Gy}$ radiation was delivered to the whole thorax of mice; 4) radiation+high dose quercetin (16 mice), 100ul quercetin $(4 \mathrm{mg} / \mathrm{ml})$ was administrated through tail vein 30 minutes before radiation, and then twice a week during the subsequent 6 weeks; 5) radiation +low dose quercetin (16 mice), mice received the same treatment as the group 5 except for low dose quercetin $(2 \mathrm{mg} / \mathrm{ml}) ; 6)$ radiation+amifostine (16 mice), 100ul amifostine $(4 \mathrm{mg} / \mathrm{ml})$ was administrated through tail vein 30 minutes before mock radiation, and then twice a week during the subsequent 6 weeks.

\section{Specimens' preparation}

In $1 \mathrm{w}, 2 \mathrm{w}, 3 \mathrm{w}, 4 \mathrm{w}, 5 \mathrm{w}, 6 \mathrm{w}, 8 \mathrm{w}$ after irradiation, one or two mice from each group were killed under anesthesia. The right side lung tissues were removed and placed in $10 \%$ formalin for 48 hours for fixation. Fixed lung tissues were embedded in paraffin and sections of $5 \mu \mathrm{m}$ thick were cut and placed on slides in preparation for histopathology and immunohistochemistry studies. The left side lung tissues were frozen and saved in $-70^{\circ} \mathrm{C}$ before use.

\section{Western blot analysis}

Western blot analysis was performed to detect the protein expression in lung tissues after irradiation. Lung tissues at indicated time were homogenized in SDS lysis buffer containing protease inhibitors on ice for $5 \mathrm{~min}$. Cell debris was removed by centrifugation at $12000 \mathrm{~g}$ for $5 \mathrm{~min}$ at $4^{\circ} \mathrm{C}$. Equal amounts of lysate protein were incubated at $100^{\circ} \mathrm{C}$ for $10 \mathrm{~min}$ and fractionated by sodium dodecylsulfonate (SDS)-polyacrylamide gel electrophoresis at $100 \mathrm{~V}$ for $90 \mathrm{~min}$ at room temperature. The separated proteins were transferred to nitrocellulose membranes, blocked with $5 \%$ defatted milk for $45 \mathrm{~min}$, and probed with primary antibodies for $2 \mathrm{~h}$ at room temperature. Membranes were washed with TBS containing Tween-20 (TBS-T), and then horseradish peroxidase-conjugated anti-rabbit secondary antibody was added for $60 \mathrm{~min}$. Immunoblots were visualized by chemiluminescence using a chemiluminescence kit (Millipore, USA) and the specific bands were recorded on $\mathrm{X}$-ray film. $\beta$-actin protein levels were used as a control to verify equal protein loading.

\section{Immunohistochemistry (IHC)}

Immunohistochemistry was performed to detect the protein expression in lung tissue slice after irradiation. level of NF- $\varkappa$ BP65, I $\varkappa$ B- $\alpha$, P38, SAPK-JNK and P44/ $\mathrm{P} 42$ protein expression were assessed with Streptavidinperoxidase methodology. IHC was carried out according to instructions of the IHC kit. IHC slides were subjected to blinded evaluation by two investigators.

\section{Grading criteria of pneumonitis and scoring criteria of IHC}

Grading criteria of pneumonitis was classified into 4 grades using light microscopy: grade 0 refers to no pneumonitis; grade 1 refers to mild pneumonitis, of which alveolar septa were thickened to an extent less than $20 \%$, and structures of alveoli were normal; grade 2 refers to medium pneumonitis, of which alveolar septa are thickened to an extent between $20 \%$ and 50\%; grade 3 refers to severe pneumonitis, of which alveolar septa are thickened to an extent over $50 \%$, and alveolar consolidation because of mononuclear leukocytes infiltration or hemorrhage is also occasional.

For IHC, cells with cytoplasmic and/or nuclear staining were defined as positive cells. The dominant of staining intensity was scored as: $0=$ negative, $1=$ weak, $2=$ intermediate, $3=$ strong. The percentage of positive cells was scored as: $0=0-5 \%, 1=6-25 \%, 2=26-50 \%, 3=51 \%$ $100 \%$. Scores of IHC $=$ scores of staining intensity $\times$ scores of percentage of positive cells.

\section{Statistical methods}

Statistical analysis were performed using the statistical package SPSS, version 18.

Kruskal-Wallis Test was used to compare the difference of RILI among groups. Further comparative analysis between any two groups used mann-whitney test. When comparisons were made between the means of two groups, a two-tailed Student's t-test was used, $p<0.05$. For multiple comparisons an ANOVA was used. Significance was defined as $p<0.05$

\section{Results}

\section{Histology change in lung tissue slide after radiation}

Hematoxylin and eosin stain was performed on the lung tissue slide to determine the histology change after irradiation. Considerable acute lung damage was noted 
at $4 \mathrm{wks}$ following exposure to $10 \mathrm{~Gy}$. Among the four groups received irradiation, radiation control exhibited the most severe acute inflammation, characterized by trachea and perivascular inflammatory cell infiltration, thicken of blood vessels and bronchial wall, edema and hyperemia in the alveolar wall and/or air spaces, and even atresia of certain pulmonary alveolus. Figure 1 showed the representative appearance of the morphological changes in the lung injured at $4 \mathrm{wks}$ after irradiation. Light microscope examination of HE slice showed that the lung tissue in irradiated mice lost their normal cord-like structure and developed extensive swelling and large fat droplets, with balloon-like changes and serious necrosis. Inflammation cells like lymphocytes and neutrophil infiltration were also observed.

Quercetin (high dose or low dose) +radiation group presented reduced alveolar inflammation compared with the irradiated group during the experiment $(\mathrm{P}<0.001$, $\mathrm{P}<0.001)$. No gradeIII RILI was observed in the high dose quercetin+radiation group. Only 4 grade IIand III RILI were shown in the low dose quercetin+radiation group. In contrast, grade IIand III RILI in radiation control group reached up to 15 mice. What's more, no significant difference was found between Quercetin (high dose or low dose)+radiation group and amifostine+radiation group ( $\mathrm{P}=0.591)$. Low dose quercetin exhibited similar radioprotective effect compared with high dose quercetin $(\mathrm{P}=0.799)$. Detailed incidence of RILI among the 80 animals was showed in Table 1.

IHC Analysis of Intracellular localization of proteins correlated with NF-kB and MAPK pathway in the Irradiated Mice Lung.

IHC was performed to detect the NF- $x$ Bp65, I $x \mathrm{~B}-\alpha$, P38, SAPK/JNK and P44/P42 expression on lung tissue slide. Cells with cytoplasmic and/or nuclear staining were defined as positive cells.

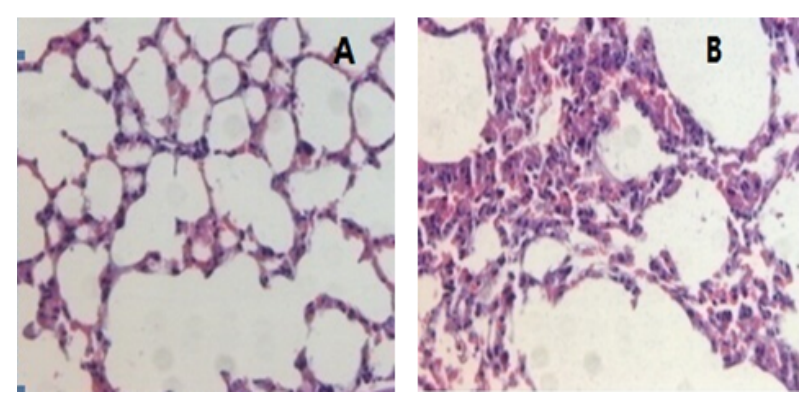

Figure 1. Morphological Changes in the Lung of Mice Injured by Radiation (HE, x400). The sections were stained with HE. A) Normal control; B) Radiation control

Table 1. Grading of Radiation Induced Lung Injury from Different Groups

\begin{tabular}{lrrrrr}
\hline Groups & $\mathrm{n}$ & \multicolumn{4}{c}{ Grade } \\
& & 0 & 1 & 2 & 3 \\
\hline Radiation control & 16 & 0 & 1 & 9 & 6 \\
Radiation+low dose quercetin & 16 & 5 & 7 & 3 & 1 \\
Radiation+high dose quercetin & 16 & 4 & 6 & 6 & 0 \\
Amifostine control & 16 & 4 & 5 & 5 & 2 \\
Quercetin control & 8 & 8 & 0 & 0 & 0 \\
Normal control & 8 & 8 & 0 & 0 & 0 \\
\hline
\end{tabular}

\section{$N F-\varkappa B$ pathway related proteins}

The treatment group that received both radiation and high or low dose quercetin injection exhibited a reduced effect on NF- $x$ Bp 65 expression in all experimental tissue specimens. As shown in Figure 2 and Table 2, positive expression of NF- $x$ Bp 65 was shown up as brown granules in the nucleus and (or) cytoplasm of inflammatory cells. NF- $x$ Bp 65 positive cells counted in Radiation+high/ low dose quercetin groups were significantly decreased compared with the radiation control $(\mathrm{P}<0.001, \mathrm{P}<0.001$, respectively). No obvious difference was observed between quercetin high and low groups $(\mathrm{P}=0.778)$. Expression level of NF- $x$ Bp65 in amifostine group was similar to those in quercetin+radiation group $(\mathrm{P}=0.764)$. In other words, quercetin injection, even at low dose, can inhibit the overexpression of NF- $x$ Bp65 induced by X-ray, high dose quercetin did not show any advantage over low dose. As for I $\chi$ B- $\alpha$, positive expression was shown up in cytoplasm as brown granules. The positive cells in radiation+ quercetin high/low doses increased compared with radiation control $(\mathrm{P}<0.01)$.

There was no statistically significant difference between radiation+quercetin and amifostine group $(\mathrm{P}=0.305)$. Therefore, we may safely draw the conclusion that quercetin may promote $\mathrm{I} x \mathrm{~B}-\alpha$ expression in

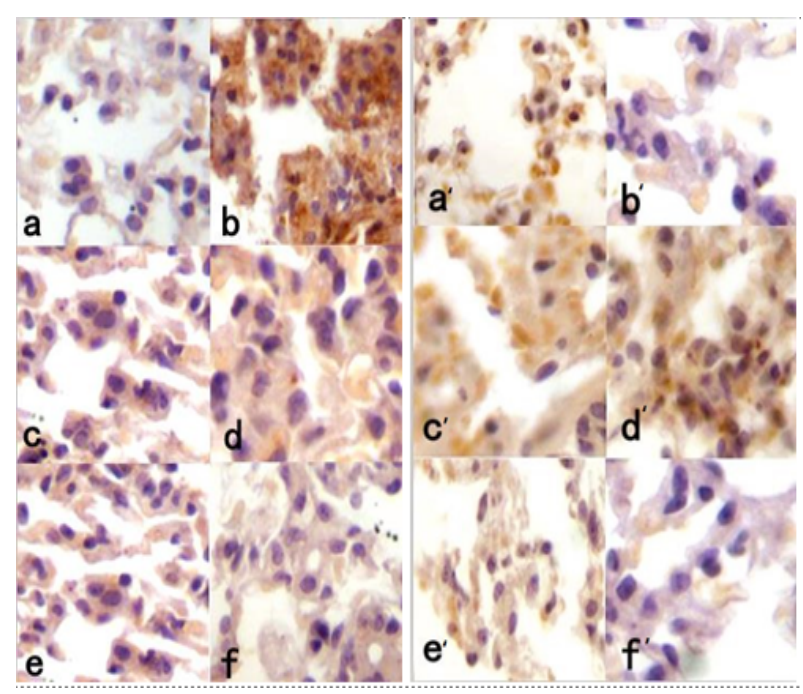

Figure 2. Immunohistochemical Staining of NF- $x \mathrm{Bp} 65$ and $\mathbf{I} \chi \mathbf{B}-\boldsymbol{\alpha}$ in Irradiated Lung Tissue. 4 weeks after irradiation. a-f and a'-f' represented NF- $x$ Bp65 and I $x$ B- $\alpha$ expression respectively. a and a': normal control; b and b': radiation control; $c$ and c': radiation+low dose quercetin injection control; $\mathrm{d}$ and d': radiation+high dosequercetin injection control; e and e': amifostine control; f and f':quercetin control

Table 2. NF- $\varkappa$ Bp65 and I $\varkappa$ B- $\alpha$ Positive Cells Counted in Different Groups $(\overline{\mathbf{x}} \pm \mathbf{S})$

\begin{tabular}{lcll}
\hline Groups & $\mathrm{n}$ & $\mathrm{NF}-\varkappa \mathrm{Bp} 65$ & $\mathrm{I} \varkappa \mathrm{B}-\alpha$ \\
\hline Radiation control & 16 & $2.38 \pm 0.65^{\mathrm{a}}$ & $1.93 \pm 0.19^{\mathrm{a}}$ \\
Radiation+low dose quercetin & 16 & $1.32 \pm 0.74^{\mathrm{b}}$ & $2.06 \pm 0.12^{\mathrm{b}}$ \\
Radiation+high dose quercetin & 16 & $1.38 \pm 0.8 \mathrm{~b}$ & $1.6 \pm 0.14 \mathrm{~b}$ \\
Amifostine control & 16 & $1.45 \pm 0.87^{\mathrm{b}}$ & $1.34 \pm 0.24^{\mathrm{b}}$ \\
Quercetin control & 8 & $0.41 \pm 0.24$ & $2.19 \pm 0.19$ \\
Normal control & 8 & $0.52 \pm 0.34$ & $2.44 \pm 0.13$ \\
\hline
\end{tabular}

*VS normal control, aP $<0.01$; VS radiation control, $\mathrm{bP}<0.05$ 


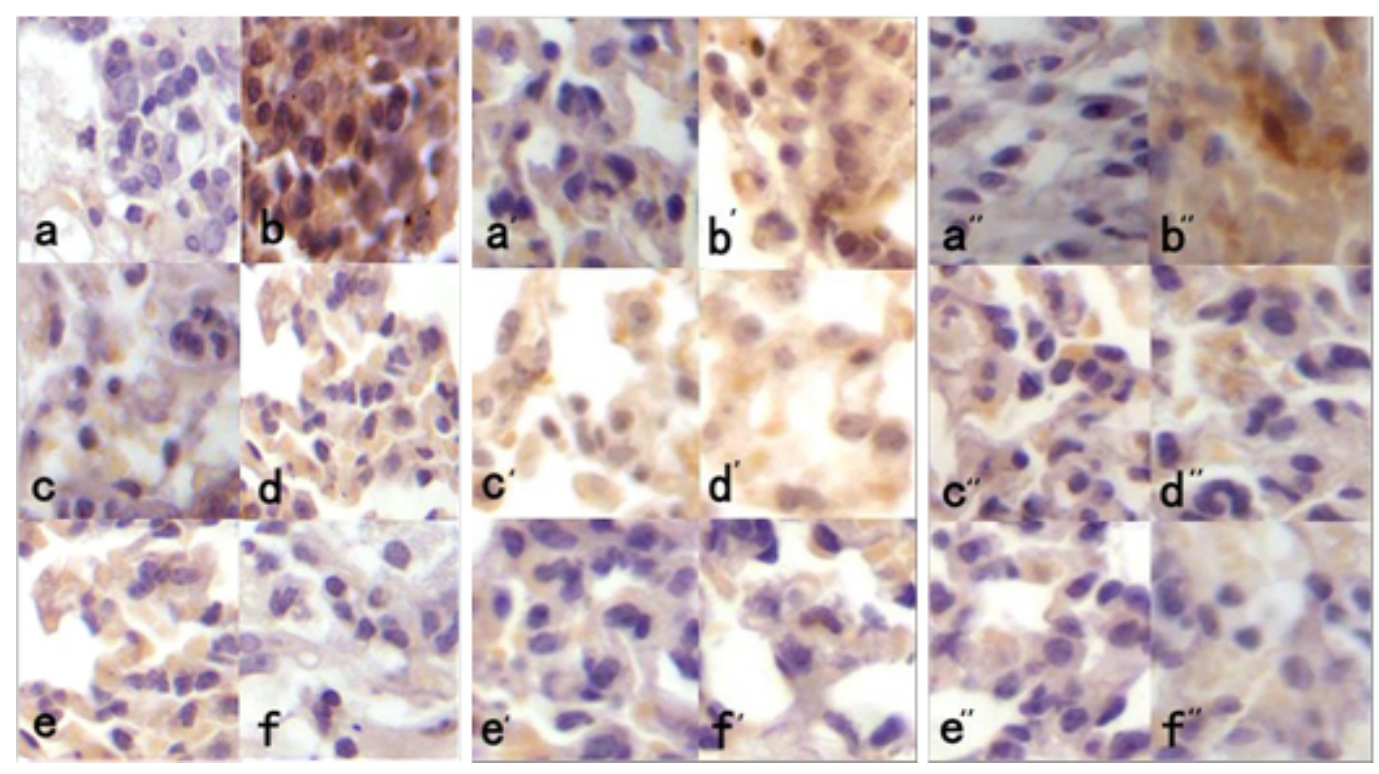

Figure 3. Immunohistochemical Staining of SAPK/JNK, p38 and p44/p42 in Irradiated Lung Tissue. 4 weeks after irradiation. a-f, a'-f' and a"-f"'represented SAPK/JNK, p38 and p44/p42 expression respectively. a, a' and a": normal control; b, b' and b": radiation control; c, c' and c": radiation + low dose quercetin injection control; d, d' and d": radiation + high dose quercetin injection control; e, e' and e": amifostine control; f, f' and f": quercetin control

Table 3. SAPK/JNK, p38 and p44/p42 Positive Cells Counted in Different Groups $(\overline{\mathbf{x}} \pm$ S)

\begin{tabular}{lccll}
\hline Groups & $\mathrm{n}$ & $\mathrm{SAPK} / \mathrm{JNK}$ & $\mathrm{p} 38$ & $\mathrm{p} 44 / \mathrm{p} 42$ \\
\hline Radiation control & 16 & $0.42 \pm 0.02^{\triangleright}$ & $0.55 \pm 0.08^{\triangle}$ & $4.5 \pm 0.20^{\triangle}$ \\
Radiation+low dose quercetin & 16 & $0.37 \pm 0.04^{*}$ & $0.24 \pm 0.03^{*}$ & $3.00 \pm 0.72^{*}$ \\
Radiation+high dose quercetin & 16 & $0.40 \pm 0.03^{*}$ & $0.3 \pm 0.12^{*}$ & $3.17 \pm 0.49^{*}$ \\
Amifostine control & 16 & $0.36 \pm 0.03^{*}$ & $0.3 \pm 0.04^{*}$ & $3.36 \pm 0.38^{*}$ \\
Quercetin control & 8 & $0.18 \pm 0.02$ & $0.12 \pm 0.01$ & $2.42 \pm 0.04$ \\
Normal control & 8 & $0.17 \pm 0.02$ & $0.13 \pm 0.02$ & $2.49 \pm 0.26$ \\
42VS radiation control, $* \mathrm{P}<0.01$; VS normal control, ${ }^{\triangle} \mathrm{P}<0.05$ & & \\
\hline
\end{tabular}

inflammatory cells.

\section{MAPK pathway related proteins}

Expression of phosphorylated JNK/SAPK, p38 and $\mathrm{p} 44 / \mathrm{p} 42$ were also tested by IHC. Positive cells manifested as brown granules in the nucleus and (or) cytoplasm of inflammatory cells. Results did not show significant difference of the proteins expression level between quercetin control and normal control. However, number of JNK/SAPK, p38 and p44/p42 positive inflammatory cells were consistently markedly increased in radiation control over amifostine $(\mathrm{P}<0.01)$, high/low dose quercetin+radiation $(\mathrm{P}<0.05, \mathrm{P}<0.05$, respectively $)$ and normal control $(\mathrm{P}<0.01)$. What's more, staining of positive inflammatory cells in radiation control was darker than the other groups (Figure 3 and Table 3 ). These results showed quercetin may play a part in repressing the over-expression of SAPK/JNK, p38 and p44/p42 induced by radiation. Furthermore, the inhibition of quercetin showed to be on par with amifostine because the number of positive cells was similar between quarcetin+radiation and amifostine+radiation group $(\mathrm{P}>0.05)$.

Western blot was used to quantitatively detect the protein expression in lung tissue after irradiation. The expression levels of the targeted proteins from NF- $x \mathrm{~B}$ and MAPK pathway were evaluated according to the ratio of the grey value of the targeted and internal proteins. Expression levels of NF- $x$ Bp65, I $x$ B- $\alpha$, JNK/SAPK,

NF-кB p65
Iкb- $\alpha$
SAPK/JNK
p38
p44/p42
$\beta-a c t i n$
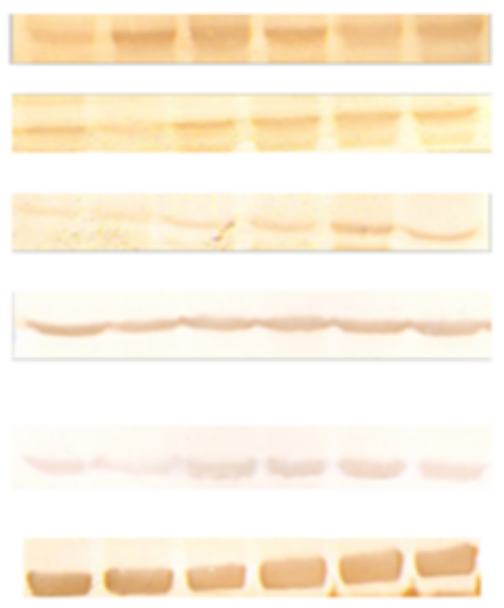

A B C D E F

Figure 4. NF- $\varkappa$ Bp65, I $\nsim$ B- $\alpha$, SAPK/JNK, p38 and p44/ p42 Expression Detected by Western Blot

p38 and p44/p42 were shown in Figure4. NF- $x$ Bp65 expression in normal control and quercetin control group were relatively small and the difference between them was not statistically significant $(\mathrm{P}=0.710)$. The level of $\mathrm{NF}-x \mathrm{Bp} 65$ increased largely in radiation control compared with normal control $(\mathrm{P}<0.01)$. However, NF- $x$ Bp65 protein expression in radiation+ quercetin (high or low 


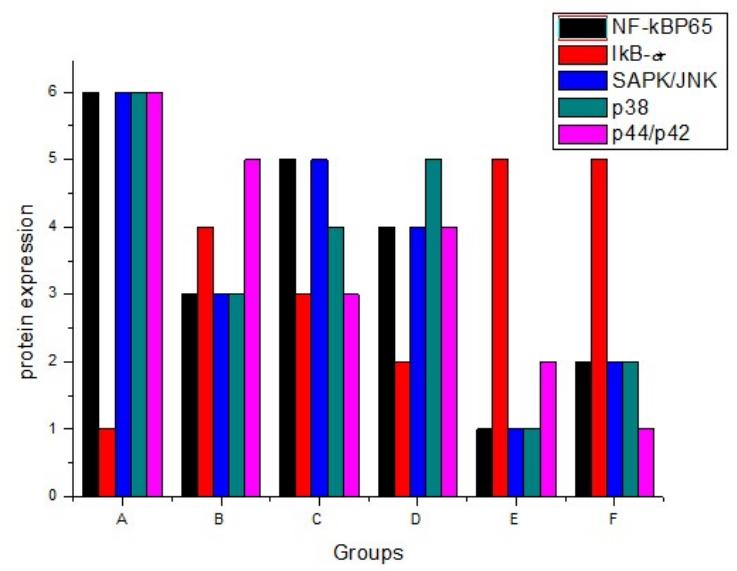

Figure 5. Relative Proteins Expression in Lung Tissue After Irradiation Detected by WB. A: Radiation control; B: radiation+low dose quercetin injection; C: radiation + high dose quercetin injection; D: amifostine control; E: quercetin control; F: normal control

dose) was much less than radiation control $(\mathrm{P}<0.05)$. Opposite results were shown in terms of expression of $\mathrm{I} \chi \mathrm{B}-\alpha$. Level of $\mathrm{I} \chi \mathrm{B}-\alpha$ in quercetin high or low group increased compared with Radiation control $(p<0.05)$.

Small amount SAPK/JNK, p38 and p44/p42 were detected in radiation control and quercetin control. Expression of these three proteins increased in radiation control compared with normal control $(p<0.05)$, high or low dose quercetin+radiation groups

$(p<0.05)$. There was no significant difference of these five proteins between quercetin high or low dose and amifostine group (Figure5).

\section{Discussion}

RILI is one of the major side effects of radiation therapy for lung carcinoma with high incidence, which not only limits the dose escalation of RT but also affects the life quality of patients, so it's greatly concerned. Recent study reported NF- $x \mathrm{~B}$ could be activated following UVB exposure on corneal epithelial cells (Lee et al., 2005; Alexander et al., 2006). NF- $\varkappa$ B controls the expression of genes encoding the pro-inflammatory cytokines, adhesion molecules, growth factors, some of the acute phase proteins, et al, all of which play critical roles in controlling most inflammatory processes (Nam et al., 2006). Ionizing radiation has been shown to activate all 3 MAPKs: extracellular signal-regulated kinase (ERK1/2), c-Jun N-terminal kinase (JNK), and p38 MAPK (Turjanski et al., 2007; Munshi et al., 2013). Activation of MAPK also contributes to inflammation. Taken together, suppression of inflammatory related cell signaling pathway such as $\mathrm{NF}-x \mathrm{~B}$ and MAPK in response to ionizing radiation is a promising strategy for improving the treatment of RILI.

The flavonoids are naturally occurring diphenylpropanoids, composed of quercetin, luteolin, kaempferol, apigenin and genistein and possess an antiinflmmatory profile (Hertog et al., 1993). Our previous study showed administration of total flavonoids might be efficacious in the prevention of radiation-induced lung damage, in line with earleir evidence (Kma, 2014)..
However, the role of diverse monomers from flavonoids and its related molecular mechanisms have not explored.

Quercetin, a member of the flavonoids family, is one of the most prominent dietary antioxidants. A recent study reported the antioxidant and anti-inflammatory properties of quercetin in two humanized models of cardiovascular disease (Kleemann et al., 2011) Treatment of macrophages with quercetin attenuated the basal expression of inflammatory genes, such as TNF- a, IL-6, IL-8, IL-1 b, interferon- g-inducible protein-10, and cyclooxygenase (COX)-2 (Overman et al., 2014). To determine whether quercetin may protect normal lung tissue from radiation and its underlying mechanism, we designed the present study. Results showed low dose quercetin injection could reduce the severity of RILI. IHC and WB verified that anti-inflammation effect of quercetin was accompanied with changes of protein expression from both NF- $x \mathrm{~B}$ and MAPK pathway, which suggested that quercetin may play the role of inhibitor of NF- $x$ B and MAPK signal transduction pathways stimulated by ionizing radiation. Therefore, we hypothesized that quercetin suppressed the NF- $x \mathrm{~B}$ activation by inhibiting the degradation of $\mathrm{I} \varkappa \mathrm{B}-\alpha$ and nuclear translocation of $\mathrm{p} 65$ subunit of NF$x \mathrm{~B}$ and attenuated phosphorylation of ERK, JNK, and p38 MAPKs. From this, Quercetin can be considered as a effective inhibitor of radiation induced inflammation manifested as RILI.

However, several critical points must be resolved before developing a potential clinical use of the quercetin: pharmacokinetic studies are needed to determine the best injection dose, which necessarily has good anti-RILI effect and low-toxicity; As a monomer of total flavonoids, quercetin alone could be efficient in treating RILI. However, roles of the rest monomers of total flavonoids and their interaction are still unknown. The present study implied that quercetin attenuated RILI through inhibiting inflammatory protein expression of NF- $\varkappa \mathrm{B}$ and MAPK signaling pathway, however, genetic changes accompanied with the biological process of quercetin injection after radiation are still unclear. Therefore, further investigation for the role of quecertin and the rest monomers is greatly needed.

\section{References}

Alexander G, Carlsen H, Blomhoff R (2006). Corneal NFkappaB activity is necessary for the retention of transparency in the cornea of UV-B-exposed transgenic reporter mice. Exp Eye Res, 82, 700-9.

Chirumbolo S (2010). The role of quercetin, flavonols and flavones in modulating inflammatory cell function. Inflamm Allergy Drug Targets, 9, 263-85.

Das DK, Chakraborty A, Sinha M, et al (2013). Modulatory role of quercetin against gamma radiation-mediated biochemical and morphological alterations of red blood cells. Int J Radiat Biol, 89, 471-81.

Hertog MG, Hollman PC, Katan MB, Kromhout D (1993). Intake of potentially anticarcinogenic flavonoids and their determinants in adults in The Netherlands. Nutr Cancer, 20, 21-9.

Horton JA, Li F, Chung EJ, et al (2013). Quercetin inhibits radiation-induced skin fibrosis. Radiat Res, 180, 205-15. 
Juan Wang et al

Kleemann R, Verschuren L, Morrison M, et al (2011). Antiinflammatory, anti-proliferative and anti-atherosclerotic effects of quercetin in human in vitro and in vivo models. Atherosclerosis, 218, 44-52.

Kma L (2014). Plant extracts and plant-derived compounds: promising players in a countermeasure strategy against radiological exposure. Asian Pac J Cancer Prev, 15, 240525.

Lee DH, Kim JK, Joo CK (2005). Translocation of nuclear factorkappaB on corneal epithelial cells induced by ultraviolet B irradiation. Ophthalmic Res, 37, 83-88.

Materska M, Konopacka M, Rogolinski J, Slosarek K (2015). Antioxidant activity and protective effects against oxidative damage of human cells induced by $\mathrm{X}$-radiation of phenolic glycosides isolated from pepper fruits Capsicum annuum $\mathrm{L}$. Food Chem, 168, 546-53.

Mehta V (2005). Radiation pneumonitis and pulmonary fibrosis in non-small-cell lung cancer: pulmonary function, prediction, and prevention. Int J Radiat Oncol Biol Phys, 63, 5-24.

Munshi A, Ramesh R (2013). Mitogen-activated protein kinases and their role in radiation response. Genes Cancer, 4, 401-8.

Nam NH (2006). Naturally occurring NF-kappaB inhibitors. Mini Rev Med Chem, 6, 945-51.

Overman A, Chuang CC, McIntosh M (2011). Quercetin attenuates inflammation in human macrophages and adipocytes exposed to macrophage-conditioned media. Int J Obes, 35, 1165-72.

Ozyurt H, Cevik O, Ozgen Z, et al (2014). Quercetin protects radiation-induced DNA damage and apoptosis in kidney and bladder tissues of rats. Free Radic Res, 48, 1247-55.

Reddy DB, Reddanna P (2009). Chebulagic acid (CA) attenuates LPS-induced inflammation by suppressing NF-kappaB and MAPK activation in RAW 264.7 macrophages. Biochem Biophys Res Commun, 381, 112-7.

Turjanski AG, Vaque JP, Gutkind JS (2007). MAP kinases and the control of nuclear events. Oncogene, 26, 3240-53.

Wang J, Xu H-W, Li B-S, Zhang J, Cheng J (2012). Preliminary study of protective effects of flavonoids against radiationinduced lung injury in mice. Asian Pac J Cancer Prev, 13, 6441-6.

Ward PA, Hunninghake GW (1998). Lung inflammation and fibrosis. Am J Respir Crit Care Med, 157, 123-9. 